\title{
Mean to long-term clinical and radiological results of unlinked total elbow arthroplasty
}

\begin{abstract}
Background \& aims: Total elbow arthroplasty (TEA) is a commonly used treatment for severely destroyed elbows in rheumatoid arthritis (RA). Indications for TEAs also include trauma and osteoarthritis. The aim of this study was to evaluate long-term clinical and radiological results of three different unlinked total elbow prostheses.
\end{abstract}

Materials and methods: We analyzed the results of 53 unlinked prostheses in 48 patients with rheumatoid arthritis placed in two Finnish hospitals between 1997 and 2007. The mean follow-up time was 15.8 years. For clinical results, we used the Mayo Elbow Performance Score (MEPS) and range of motion (ROM). For radiological results, we used pre- and postoperative radiographs. For calculating the survival time, we used the Kaplan-Meyer survival analysis.

Results: MEPS scores were 30 points higher postoperatively and stayed level during the follow-up. Improvements in ROM were also significant. Active extension motion deficiency was 6 degrees lower, active flexion improved by 10 degrees, forearm pronation improved by 8 degrees and supination improved by 8 . Radiological loosening was very common in both components. The complication rate was $28 \%$ and revision surgery was needed with $23 \%$ of the patients. For implant survival, we found a mean survival time of 70 months.

Conclusion: Unlinked total elbow arthroplasties provide excellent long-term results for improving elbow functionality and significantly increase the quality of life, but complication and revision rates are high. Compared to other common arthroplasties such as knee and hip arthroplasties TEAs have much more complications and much shorter survival times. Further development of TEAs is needed to decrease complication and revision rates.

Keywords: arthroplasty, elbow, orthopaedic surgery, prosthesis, rheumatoid arthritis, unlinked

\section{Introduction}

The first commercial total elbow arthroplasty (TEA) was introduced in 1972 for rheumatoid arthritis. ${ }^{1}$ While both prostheses and surgical techniques have evolved over the last 45 years, the basic principle of function have remained the same. More developed models and surgical techniques have made it possible to use these implants also in elbow traumas and osteoarthritis. Despite new indications, the most common patient is 60 years old woman with rheumatoid arthritis. ${ }^{2}$ Previous studies have shown that TEAs significantly improve function, the range of motion and stability of the elbow and greatly lessens the pain of elbow. ${ }^{3}$

There are two types of prostheses in use: Semi-constrained and unlinked. In semi-constrained models, implants are mechanically linked with so-called sloppy-hinge. In unlinked models, the implants are not mechanically linked together, and the stability is provided by the support of the patient's own tissue. ${ }^{4}$ To provide enough support to prevent dislocation the patient's bones and ligaments must be sufficiently well preserved. Also, the function of elbow's flexor and extensor muscles must be sufficient enough., ${ }^{5,6}$ With unlinked prostheses the movement of the elbow is almost the same as in normal elbow and in theory, wear out less and reduce the risk for loosening and osteolysis. ${ }^{5,7}$ Although the most common complications are the same with both types of prostheses in practice it has been shown that unlinked prostheses are more likely to develop complications than semi-constrained prostheses. The most common complications are clinical and radiological loosening, periprosthetic fractures, instability of the prosthesis, infection and mechanical failure. ${ }^{2,3,8,9}$
Volume II Issue I - 2019

\author{
A Hyytiäinen, ' H Tiusanen, ${ }^{2}$ P Sarantsin, ${ }^{2}$ M \\ Stenholm ${ }^{2}$ \\ 'University of Turku, Clinical Department, Department of \\ Orthopaedics and Traumatology, Finland \\ ${ }^{2}$ TYKS Orto, Department of Rheumaorthopaedic Surgery, Turku \\ University Hospital and University of Turku, Finland
}

Correspondence: A Hyytiäinen, University of Turku, Clinical Department, Department of Orthopaedics and Traumatology, PO Box 282070 I Turku, Finland, Tel +358405489293. Email alemhy@utu.fi

Received: February 05, 2019 | Published: February 12, 2019
Radiolucency and most complications can be detected in radiographs. Radiolucent lines less than one millimeter wide are accepted. During time in some cases radiolucent lines widen. With most patients these widen radiolucent lines doesn't progress and the component doesn't become radiologically loose. In some cases, the widening of radiolucent lines starts to progress. Radiolucent line wider than two millimeters indicates radiological loosening. All radiologically loose prostheses don't become clinically loose. ${ }^{10}$ Osteolysis is a common phenomenon in arthroplasties and can be seen in radiographs as areas of radiolucency in the bone near the prostheses. Osteolysis can lead to loosening or periprosthetic fracture. Luxation, subluxation and polyethylene wear can be seen in radiographs as malposition of the joint. The aim of this study was to analyze the Mean to long-term clinical and radiological results of total elbow arthroplasties with three types of unlinked prostheses.

\section{Materials and methods}

From 1997 to 2007, a total of 53 TEAs was performed for 48 patients with rheumatoid arthritis in two hospitals using 19 IBP (Biomet), 30 Kudo (Biomet) and 4 Souter-Strathclyde (Stryker) unlinked prostheses. There were 42 women and 6 men. The mean age of patients was 61 (range 35-86) years. Patients were evaluated preoperatively, postoperatively and at $1,3,5,7,10,15$, and 17 years, if available. The mean follow-up of patients was 15.8 (range 3.8-20.8) years. 19 patients were lost because of death unrelated to the prostheses during the follow-up. Some of patients had undergone previous surgeries for elbow. Previous operations were either synovectomy or excision of capitulum radii which was common operation in that time in Finland. 
Previous operations have been excluded from this study because they didn't have effect on outcome. The data was gathered from electronic patient records. Demographics are presented in Table 1.

Clinical evaluation was made by standardized protocol by an independent observer preoperatively, postoperatively and during follow-up. Clinical evaluation of elbow's function was made by using the Mayo Clinic Performance Scoring ${ }^{11}$ (MEPS) and ranges of motion (ROM) of elbow's extension and flexion and forearms pronation and supination. At follow-ups patients were also interviewed for subjective improvement of function and overall satisfaction of the results with a scale of 1 to 4 , where 1 was worse than preoperatively, 2 was no effect, 3 was slightly better and 4 was much better than preoperatively.

Preoperative radiological evaluation of elbows was made by using Larsen's grading. ${ }^{12}$ In the scale for 1 to 5 , all the patients had a degree of destruction from level 3 to 5 or from medium destructive abnormalities to severe mutilation. The average Larsen's grade was 4.0 while the range was from 3 to 5 . Postoperative radiological results were evaluated by searching radiolucency lines from radiographs. Ulnar and humeral components were both divided into four areas to define the location of radiolucent lines (Figure 1: Areas for radiolucency). Preoperative information is presented in Table 1.

The elbow prostheses were chosen for treatment because it is the number one choice for mutilated elbows in Finland. Unlinked prostheses were chosen because they were commonly used in Finland in that time for patients with RA, later semi linked prostheses have taken their place. The medical treatment of RA has varied highly during the follow-up; therefore, medication has been excluded from the study. In Finland the treatment of RA begins with combination therapy ${ }^{13}$ and other drugs are added afterwards if needed. In Finland biological drugs have been available for treatment of RA only since early 2000 s.

Table I Demographics and Clinical Results

\begin{tabular}{|c|c|c|c|c|}
\hline Number of patients & 53 & & & \\
\hline \multicolumn{5}{|l|}{ Sex, n (\%) } \\
\hline Males & \multicolumn{4}{|l|}{$42(88 \%)$} \\
\hline Females & \multicolumn{4}{|l|}{$6(12 \%)$} \\
\hline Mean age at operation (years) & \multicolumn{4}{|l|}{61} \\
\hline Mean follow-up (years) & \multicolumn{4}{|l|}{15.8} \\
\hline Mean Preoperative Larsen's Grade & \multicolumn{4}{|l|}{4} \\
\hline \multicolumn{5}{|l|}{ Type of Prosthesis, n (\%) } \\
\hline IBP & \multicolumn{4}{|l|}{$19(36 \%)$} \\
\hline Kudo & \multicolumn{4}{|l|}{$30(57 \%)$} \\
\hline \multirow[t]{2}{*}{ Souter-Strathclyde } & \multicolumn{4}{|l|}{$4(7 \%)$} \\
\hline & Preoperative Mean & Postoperative Mean & Improvement & $\mathrm{P}$ \\
\hline MEPS & $56(15-100)$ & $86(35-100)$ & $30(23-36)$ & $<0.0001$ \\
\hline \multicolumn{5}{|l|}{ ROM (degrees) } \\
\hline Extension deficiency & $30(10-70)$ & $24(0-60)$ & $6(2-10)$ & $<0.002$ \\
\hline Flexion & $127(75-150)$ & $137(105-155)$ & $10(6-14)$ & $<0.001$ \\
\hline Pronation & $73(30-95)$ & $80(30-100)$ & $8(2-13)$ & $<0.005$ \\
\hline Supination & $67(5-100)$ & $74(10-100)$ & $8(2-14)$ & $<0.007$ \\
\hline
\end{tabular}

MEPS, Mayo Clinic Elbow Performance Score; ROM, Ranges of motion

\section{Statistical methods}

For calculating implant survival rates Kaplan-Meyer survival analysis was used while revision surgery was the endpoint. Differences between preoperative and postoperative of clinical results were compared with Paired-Samples T-test and considered significant if the $P$ value was $<0.05$ on a 2-tailed test. SPSS Statistics software version 20.5 (IBM SPSS Statistics 25) was used for statistical analysis.

\section{Results}

Clinical results are presented in Table 2. The MEPS scores improved significantly after the operation and remained at the same levels in the follow-up. In this study, the improvement of MEPS after the operation was 30 points $(95 \%$ confidence interval (CI), 23-36,
$\mathrm{P}<0.0001, \mathrm{~N}=50)$. The preoperative mean of MEPS was $56(15-100)$ and postoperatively the mean was $86(35-100)$. No patient had worse MEPS postoperatively than preoperatively.

MEPS scores were also evaluated during the follow-up. In the follow-up means were: $95(45-100, \mathrm{~N}=47)$ after 1 year, $91(35-100$, $\mathrm{N}=41)$ after 3 years, $96(65-100, \mathrm{~N}=35)$ after 5 years, $88(40-100$, $\mathrm{N}=18)$ after 7 years, $86(100-60, \mathrm{~N}=15)$ after 10 years, $85(100-65$, $\mathrm{N}=4)$ after 15 years and $80(\mathrm{~N}=1)$ after 17 years (Figure 2: The mean MEPS during follow up). The ranges of motion of the elbow and the forearm were also significantly higher postoperatively in this study. Active extension motion deficiency was 6 degrees lower $(95 \% \mathrm{CI}$, $2-10, \mathrm{P}<0.002, \mathrm{~N}=50)$. For extension deficiency, the preoperative mean was $30(10-70)$ degrees and postoperatively $24(0-60)$ degrees. 
Active flexion improved by 10 degrees $(95 \%$ CI, 6-14, $\mathrm{P}<0.001$, $\mathrm{N}=50$ ) while the preoperative mean for flexion was $127(75-150)$ degrees and the postoperative mean was 137 (105-155) degrees. Forearm pronation improved by 8 degrees $(95 \% \mathrm{CI}, 2-13, \mathrm{P}<0.005$, $\mathrm{N}=50)$. Forearm pronation was $73(30-95)$ degrees preoperatively and 80 (30-100) degrees postoperatively. Forearm supination improved by 8 degrees $(95 \% \mathrm{CI}, 2-14, \mathrm{P}<0.007, \mathrm{~N}=50)$ degrees while the means were $67(5-100)$ degrees for preoperative and $74(10-100)$ degrees for postoperative supination.

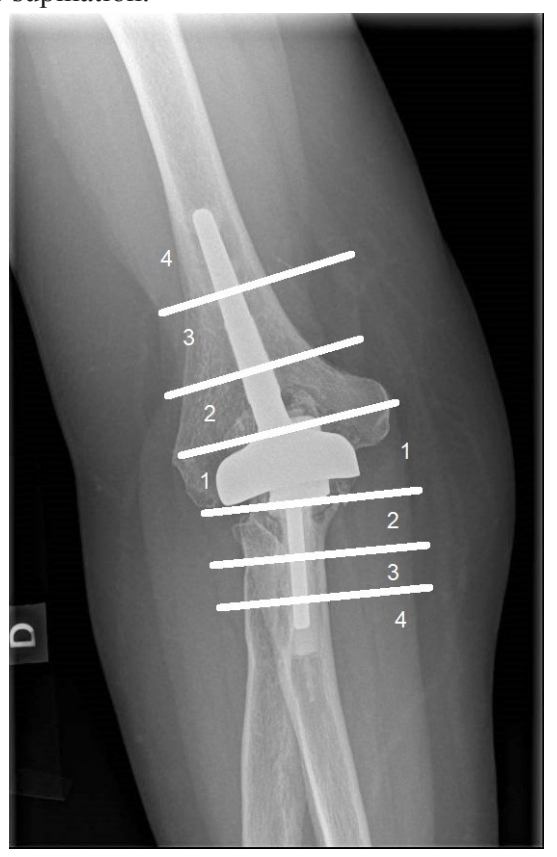

Figure I Areas of radiolucency: Ulnar and humeral components were both divided into four areas to define the location of radiolucent lines.

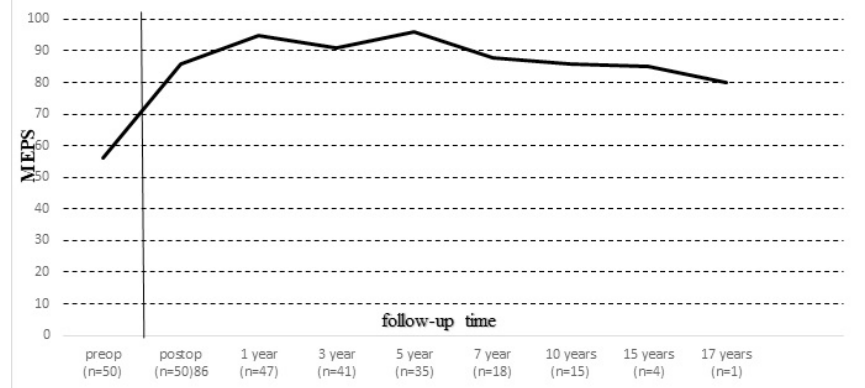

Figure 2 Mean MEPS during follow up: MEPS means evaluated during the follow up

The satisfaction and the patients' own estimation of the improvement of daily function were also very high. Most of the patients had great results even after the latest follow-up. None of the patients reported worse function than preoperatively. In the scale from 1 to 4 where 1 was worse than preoperatively, 2 was no effect, 3 was slightly better and 4 was much better than preoperatively, the mean for patient satisfaction was $3.6(2-4)$ and the mean for improvement of daily function was $3.6(2-4)$.

Radiological results are presented in Table 2. At the follow-up, study radiolucency was observed in all four areas for both components.
For ulnar component radiolucency was observed for 10 (19\%) TEAs in the 1 st area, for $3(6 \%)$ in the 2 nd area, for $2(4 \%)$ in the 3 rd area and $2(4 \%)$ in the 4 th area. For humeral component radiolucency was observed for $12(23 \%)$ TEAs in the 1st area, for $5(9 \%)$ in the 2 nd area, for $2(4 \%)$ in the 3 rd area and for $1(2 \%)$ in the 4 th area. The component was radiologically loose if radiolucency was present in 3rd or 4th area. Radiological results are presented in Table 2.

Table 2 Radiolucency, Complications and Revisions in follow-up

\begin{tabular}{lllll}
\hline Radiolucency & & & \\
\hline & $1^{\text {St }}$ area & $2^{\text {nd }}$ area & $3^{\text {rd }}$ area & $4^{\text {th }}$ area \\
Ulnar component & $10(19 \%)$ & $3(6 \%)$ & $2(4 \%)$ & $2(4 \%)$ \\
Humeral component & $12(24 \%)$ & $5(10 \%)$ & $2(4 \%)$ & $1(2 \%)$ \\
\hline $\begin{array}{l}\text { Complications and } \\
\text { revisions }\end{array}$ & Number of & Number of revisions & \\
\hline Total & complications & & 12 & \\
Aseptic Loosening & 6 & 6 & \\
Infection & 15 & 0 & \\
$\begin{array}{l}\text { Dehiscence of triceps } \\
\text { tendon }\end{array}$ & 1 & 1 & \\
Luxation & 3 & 3 & \\
Subluxation & 2 & 0 & \\
$\begin{array}{l}\text { Polyethylene wear } \\
\text { Periprosthetic }\end{array}$ & 1 & 1 & 1 & \\
fracture & & &
\end{tabular}

\section{Complications}

In this study, complications were observed in 15 (28\%) elbows. Complications included aseptic loosening of the component in 6, luxation in 3 , subluxation in 2 , dehiscence of triceps tendon in 1 , periprosthetic fracture in 1 , polyethylene wear in 1 and infection in 1 cases. Radiological loosening was observed in $8 \%$ of the ulnar and $6 \%$ of the humeral components. Revision surgery was needed in 12 cases $(23 \%)$. The causes were aseptic loosening in 6 , luxation of the elbow in 3, periprosthetic fracture in 1, dehiscence of triceps tendon in 1 and polyethylene wear in 1 cases (Table 2). With revision surgery serving as an endpoint, Kaplan-Meier survival analysis shows that unlinked TEAs have the mean survival time of 70 months (95\% CI, 47-94) (Figure 3 Kaplan-Meier survival analysis). Survival time variated between 1 to 121 months. Complications and revisions are presented in Table 2.

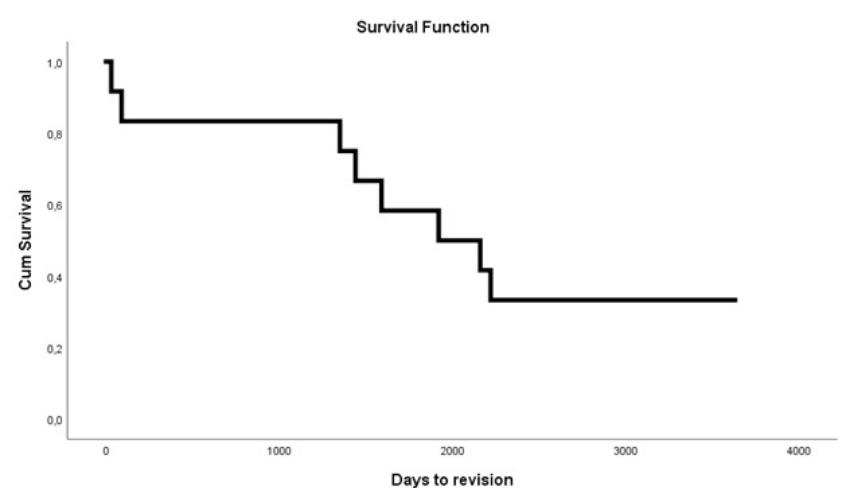

Figure 3 Kaplan-Meier survival analysis. Kaplan-Meier survival analysis for survival of prothesis, with revision surgery serving as an endpoint. 


\section{Discussion}

The limitation of our study is that it is retrospective, and the number of the prosthesis is quite low. However in our study, we found good Mean to long-term results for elbow performance. MEPS stayed at high level during follow-up time. No patient had worse MEPS postoperatively than preoperatively. MEPS were in line with other studies and ROM was slightly better in our study. ${ }^{3,4,14}$ Compared to studies for the semi-constrained prostheses, there was no difference in elbow performance between types of prostheses. ${ }^{3,15,16}$ We found also excellent results in patient satisfaction and improvement of daily function.

In our study complication rates and revision, rates were very high for the unlinked prostheses. Complication rates were exact or slightly more common in our study than in most other studies of both types of prostheses. ${ }^{3,8,17}$ All the complications that occurred in our study are common with total elbow arthroplasties despite the type of prosthesis. ${ }^{4,9,18}$ In our study, we found very high revision rate compared to most other studies. ${ }^{2,3,8}$ Two studies showed worse results than ours for complication rates and revision rates. ${ }^{4,19}$ Two early revisions, after 1 month and 3 months from primary surgery, have most likely worsened our results and most revisions occurred during years. Typical complications and indications for revision were the same in most of the studies for both types of prostheses. ${ }^{2,3,8}$ Studies show that Semi-constrained TEAs have better results for implant survival. ${ }^{2,3,8,20}$ Compared to knee and hip prostheses studies show that TEAs have much more complications and higher revision rates..$^{21,22}$

Radiolucency occurred in high percentage. Most of the patients with radiological loosening had no symptoms and radiological loosening rarely led to revision. Radiological loosening was most common in the distal end of the humeral component and the proximal end of the ulnar component. High rates of complications and revisions stay unclear. High rates might be explained trough long follow up time, cementing techniques and the quality of cement used. In other studies, the follow up time have been shorter and, in our study, the most revisions occurred towards the end of follow up, which might explain high rates. For ulnar component there are no commercial plugs, so the plugs used are made by modifying other plugs or there is no plug used at all. This may lead to unperfect cementing and may be the reason for loosening of ulnar component. Also, the condition of patients with RA have been significantly developed due advancements in medication which may distort our results compared to studies with newer patients.

\section{Conclusion}

Unlinked total elbow arthroplasties provide excellent results for improving elbow functionality and significantly increase the quality of life, but complication and revision rates are high. Semiconstrained models have better results and it is advisable to prefer semi-constrained models over unlinked models. Compared to other common arthroplasties such as knee and hip arthroplasties TEAs have much more complications and much shorter survival times. Further development of TEAs is needed to decrease complication and revision rates

\section{Conflicts of interest}

All authors declare that they have no conflict of interest.

No Funding was provided for this study.
The ethical community of our institute approved the study.

\section{References}

1. Dee R. Total replacement arthroplasty of the elbow for rheumatoid arthritis. J Bone Jt Surg Br. 1972;54(1):88-95.

2. Plaschke HC, Thillemann TM, Brorson S, et al. Implant survival after total elbow arthroplasty: A retrospective study of 324 procedures performed from 1980 to 2008. J Shoulder Elb Surg. 2014;23(6):829836.

3. Welsink CL, Lambers KTA, van Deurzen DFP, et al. Total Elbow Arthroplasty: A Systematic Review. JBJS Rev. 2017;5(7):e4.

4. Park SE, Kim JY, Cho SW, et al. Complications and revision rate compared by type of total elbow arthroplasty. J Shoulder Elb Surg. 2013;22(8):1121-1127.

5. Iwamoto T, Ikegami H, Suzuki T, et al. The History and Future of Unlinked Total Elbow Arthroplasty. Keio J Med. 2018;67(2): 19-25.

6. Mehta SS, Watts AC, Talwalkar SC, et al. Early results of Latitude primary total elbow replacement with a minimum follow-up of 2 years. J Shoulder Elb Surg. 2017;26(10):1867-1872.

7. Sanchez-Sotelo J. Total elbow arthroplasty. Open Orthop J. 2011;5:115123.

8. Voloshin I, Schippert DW, Kakar S, et al. Complications of total elbow replacement: A systematic review. J Shoulder Elb Surg. 2011;20(1):15868.

9. Gschwend N, Simmen BR, Matejovsky Z. Late complications in elbow arthroplasty. J shoulder Elb Surg. 1996;5(2 Pt 1):86-96.

10. Morrey BF, Bryan RS, Dobyns JH, et al. Total elbow arthroplasty. A five-year experience at the Mayo Clinic. J Bone Joint Surg Am. 1981;63(7):1050-1063.

11. Morrey BF, Adams RA. Semiconstrained arthroplasty for the treatment of rheumatoid arthritis of the elbow. J Bone Joint Surg Am. 1992;74(4):479-490.

12. Larsen A, Dale K, Eek M. Radiographic Evaluation of Rheumatoid Arthritis and Related Conditions by Standard Reference Films. Acta Radiol Diagn. 1977;18(4):481-491.

13. Möttönen T, Hannonen P, Leirisalo-repo M, et al. Comparison of combination therapy with single-drug therapy in early rheumatoid arthritis: a randomised trial. FIN-RACo trial group. Lancet. 1999;353(9164):1568-1573.

14. Plaschke HC, Thillemann TM, Brorson S, et al. Outcome after total elbow arthroplasty: a retrospective study of 167 procedures performed from 1981 to 2008. J Shoulder Elb Surg. 2015;24(12):1982-1990.

15. Aldridge JM, Lightdale NR, Mallon WJ, et al. Total elbow arthroplasty with the Coonrad/Coonrad-Morrey prosthesis. J Bone Joint Surg Br. 2006;88(4):509-554.

16. Giannicola G, Sacchetti FM, Antonietti G, et al. Radial head, radiocapitellar and total elbow arthroplasties: A review of recent literature. Injury. 2014;45(2):428-436.

17. Choo A, Ramsey ML. Total Elbow Arthroplasty: Current Options. J Am Acad Orthop Surg. 2013;21(7):427-437.

18. Schneeberger AG, Kösters MC, Steens W. Comparison of the subjective elbow value and the mayo elbow performance score. J Shoulder Elb Surg. 2014;23(3):308-312.

19. Throckmorton T, Zarkadas P, Sanchez-Sotelo J, et al. Failure patterns after linked semiconstrained total elbow arthroplasty for posttraumatic arthritis. J Bone Jt Surg Am. 2010;92(6):1432-1419. 
20. Levy JC, Loeb M, Chuinard C, et al. Effectiveness of revision following linked versus unlinked total elbow arthroplasty. J Shoulder Elb Surg. 2009;18(3):457-462.

21. Carr AJ, Robertsson O, Graves S, et al. Knee replacement. Lancet. 2012;379(9823):1331-1340.
22. Learmonth ID, Young C, Rorabeck C. The operation of the century: total hip replacement. Lancet. 2007;370(9597):1508-1519. 